\title{
Genetic control of plasma lipid levels in a cross derived from normoglycaemic Brown Norway and spontaneously diabetic Goto-Kakizaki rats
}

\author{
K. Argoud • S. P. Wilder • M. A. McAteer • \\ M. T. Bihoreau • F. Ouali • P. Y. Woon • R. H. Wallis • \\ A. Ktorza • D. Gauguier
}

Received: 3 March 2006 / Accepted: 20 June 2006 / Published online: 16 September 2006

(C) Springer-Verlag 2006

\begin{abstract}
Aims/hypothesis Dyslipidaemia is a main component of the insulin resistance syndrome. The inbred Goto-Kakizaki (GK) rat is a model of spontaneous type 2 diabetes and insulin resistance, which has been used to identify diabetes-related susceptibility loci in genetic crosses. The objective of our study was to test the genetic control of lipid metabolism in the GK rat and investigate a possible relationship with known genetic loci regulating glucose homeostasis in this strain.

Materials and methods Plasma concentration of triglycerides, phospholipids, total cholesterol, HDL, LDL and VLDL cholesterol were determined in a cohort of 151 hybrids of an F2 cross derived from GK and non-diabetic Brown Norway $(\mathrm{BN})$ rats. Data from the genome-wide scan of the F2 hybrids were used to test for evidence of genetic linkage to the lipid quantitative traits.

Results We identified statistically significant quantitative trait loci (QTLs) that control the level of plasma phospho-
\end{abstract}

Electronic supplementary material Supplementary material

is available for this article at http://dx.doi.org/10.1007/s00125-006-

0396- $\mathrm{z}$ and is accessible to authorised users.

K. Argoud $\cdot$ S. P. Wilder $\cdot$ M. A. McAteer $\cdot$ M. T. Bihoreau

P. Y. Woon $\cdot$ R. H. Wallis $\cdot$ D. Gauguier $(\bowtie)$

The Wellcome Trust Centre for Human Genetics,

University of Oxford,

Roosevelt Drive,

Headington, Oxford OX3 7BN, UK

e-mail: gdomi@well.ox.ac.uk

F. Ouali $\cdot$ A. Ktorza

Laboratory of Pathophysiology of Nutrition, CNRS UMR 7059,

University of Paris 7,

Paris, France

\section{A. Ktorza}

Servier International Research Institute,

Courbevoie Cedex, France lipids and triglycerides (chromosome 1), LDL cholesterol (chromosome 3) and total and HDL cholesterol (chromosomes 1 and 5). These QTLs do not coincide with previously identified diabetes susceptibility loci in a similar cross. The significance of lipid QTLs mapped to chromosomes 1 and 5 is strongly influenced by sex.

Conclusion/interpretation We established that several genetic loci control the quantitative variations of plasma lipid variables in a $\mathrm{GK} \times \mathrm{BN}$ cross. They appear to be distinct from known GK diabetes QTLs, indicating that lipid metabolism and traits directly relevant to glucose and insulin regulation are controlled by different gene variants in this strain combination.

Keywords Cholesterol · Genetics · Lipoproteins . Quantitative trait locus $\cdot$ QTL $\cdot$ Triglycerides
Abbreviations
BN Brown Norway
cM centimorgan
ESM Electronic supplementary material
GK Goto-Kakizaki
OLETF Otsuka Long-Evans Tokushima fatty
QTL quantitative trait locus
SR Dahl salt-resistant normotensive rat
SS Dahl salt-sensitive hypertensive rat
SHR spontaneously hypertensive rat
SHRSP spontaneously hypertensive stroke-prone (rat)

\section{Introduction}

Insulin resistance lies at the centre of a group of common human disorders, including type 2 diabetes mellitus, 
obesity, hypertension and dyslipidaemia and is a major risk factor for the development of cardiovascular diseases [1]. The cause of each of these disorders, which together have been referred to as the 'insulin resistance syndrome' or the 'metabolic syndrome' [2, 3], is complex and typically involves environmental and genetic risk factors [4]. Family-and population-based studies have addressed the strong contribution of genetic factors in the development of this syndrome [5]. Although independent gene variants can cause specific pathophysiological components of the metabolic syndrome, it is possible that in some cases the underlying cluster of disorders stems from the pleiotropic or sequential effects of a limited number of gene variants. Genetic investigations designed to test these hypotheses and identify causative genes are particularly difficult in humans [5]. Rodent models provide powerful experimental systems to study the relationship between phenotypes involved in the metabolic syndrome and test their genetic control [6].

The inbred Goto-Kakizaki (GK) rat strain is a model of spontaneous diabetes, primarily characterised by glucose intolerance, hyperinsulinaemia, tissue-specific insulin resistance, altered insulin secretion in vivo and in vitro, and reduced beta cell mass [7]. Evidence of salt-induced hypertension and moderately elevated plasma concentrations of cholesterol and triglycerides is also documented in this strain [8, 9]. This range of pathological features develops as a consequence of the expression of naturally occurring gene variants isolated from an outbred Wistar colony by repeated breeding of rats selected solely on the basis of glucose intolerance [10]. Genetic studies in this model have primarily aimed at investigating the inheritance of glucose intolerance and altered insulin secretion and defining regions of the GK genome that contribute to diabetes susceptibility. Independent genetic studies in F2 cohorts derived from the GK rat and normoglycaemic Brown Norway $(\mathrm{BN})$ or Fisher rats have demonstrated the polygenic control of diabetes-related phenotypes [11, 12]. At least six different quantitative trait loci (QTLs) control fasting glycaemia and insulinaemia, glucose tolerance, glucose and arginine-stimulated insulin secretion, body weight and adiposity in a $\mathrm{GK} \times \mathrm{BN}$ cross [12].

In order to test the existence of gene polymorphisms contributing to changes in lipid metabolism in this strain combination and to investigate their relationship with known diabetes-related QTLs in GK, we searched for QTLs involved in the control of plasma lipid variables in a new cohort of $\mathrm{GK} \times \mathrm{BN}$ F2 hybrids. We show that genetic loci that affect plasma concentrations of triglyceride, phospholipids and total, LDL and HDL cholesterol, and were mapped by us to rat chromosomes 1, 3 and 5 do not coincide with QTLs previously identified in the GK rat.

\section{Materials and methods}

Animals

GK rats from the colony initiated in Paris in 1989 were used. BN rats were obtained from a commercial supplier (Charles River Laboratories, Margate, UK). Rats were weaned at 21 days, had free access to water and standard laboratory chow pellets (ERB, Whitam, UK), and were maintained on a 12-h light and dark cycle. All procedures were carried out in accordance with national and institutional guidelines.

Two reciprocal F1 populations (GK females mated with $\mathrm{BN}$ males and $\mathrm{BN}$ females mated with GK males) were initially produced. A cohort of $151 \mathrm{~F} 2$ rats (76 males and 75 females) was independently derived from the two series of F1 animals, 77 originated from GK females and 74 from GK males.

Analysis of plasma lipid and glucose concentrations

At 2 months of age, BN, GK, F1 and F2 progenies were fasted overnight for 16 to $18 \mathrm{~h}$. Male and female rats of the F1 generation were randomly chosen regardless of the direction of the cross. The following morning, blood samples were collected via the tail vein into EDTA-tubes and centrifuged at $700 \times \mathrm{g}$. Plasma was collected and immediately stored at $-80^{\circ} \mathrm{C}$ until analysed. Plasma concentrations of total cholesterol, HDL-cholesterol, LDLcholesterol, triglycerides, phospholipids and glucose were determined using diagnostic enzymatic/colorimetric kits (ABX, Shefford, UK) on an automatic analyser (Cobas Mira Plus; ABX, Shefford, UK). Spleens were dissected and snap frozen for subsequent DNA preparation.

Genetic markers

In order to provide optimal genome coverage in the new F2 cross, all markers used in this study were selected in our existing linkage maps derived from a $\mathrm{GK} \times \mathrm{BN}$ F2 cross [13]. Markers were chosen so as to cover the 21 rat chromosomes with an average spacing of 10 to 15 centimorgan (cM) between adjacent loci. Marker information, including primer sequences and PCR conditions, is publicly available through our data repository (http://www.well.ox. ac.uk/rat_mapping_resources).

Genotype determination

Genomic DNA of the F2 rats was prepared from spleen samples as previously described [12]. For genotype analysis, the PCR reaction volume was $20 \mu$ containing $50 \mathrm{ng}$ of genomic DNA as previously described [14]. 
Thermocycling was initiated by $4 \mathrm{~min}$ of denaturation and followed by a touch-down protocol from 60 to $55^{\circ} \mathrm{C}$ or 55 to $50^{\circ} \mathrm{C}$. PCR products were separated by electrophoresis on agarose gels and visualised by ethidium bromide gel staining for genotype determination. Oligonucleotides were synthesised commercially by Sigma-Genosys (Pampisford, UK).

\section{Genetic map construction}

Genetic maps were constructed using the JoinMap version 2.0 suite of programs [15] as previously described [14]. Following the construction of initial genetic maps, double recombination events were identified with the jmchk module (JoinMap) and verified. Data corresponding to double recombination were verified. We considered unexplained genotypes to be those with a threshold of $>3$ for the test statistic of $\log 10(1 / \mathrm{p})$.

\section{Statistical methods and QTL analyses}

Phenotype correlation was investigated using the $R$ [16] package statistical microarray analysis. All correlations between standardised residuals were calculated and plotted. The significance of the correlations was adjusted for multiple testing using the Dunn-Šidák method [17].

Prior to QTL mapping, all phenotypes were corrected for sex by regression and the standardised residuals were investigated for departures from normality using the Kolmogorov-Smirnov and Shapiro-Wilk tests and SPSS version 12.0 (SAS Institute, Cary, NC, USA). For variables with significant departure from normality, the logarithmtransformed raw data were regressed by sex, and the standardised residuals were analysed for departures from normality. In all further linkage analyses, the standardised residuals that best fitted the normal distribution were used. We initially evaluated linkages between marker genotypes and phenotypes using automated ANOVA tests followed by permutation tests $(n=10,000)$, which were applied to calculate the threshold of significance for each pair of genetic-phenotypic marker [18]. R/qtl [19] was applied for generation of the genome-wide scans. For each of the standardised variables, Haley-Knott regression [20] was used. Subsequently, genome scans were carried out for the two sexes to test the existence of sex-related effects in QTL detection. Finally, linear regression was used to test whether the direction of cross was a statistically significant factor for each detected QTL.

MAPMAKER/QTL software was subsequently used for interval mapping [21] for chromosomes exhibiting evidence of significant genetic linkage to plasma lipid variables. Correlations between marker genotypes and phenotypes were calculated using SPSS version 12.0 (SAS).

\section{Results}

Strain-specific pattern of glycaemia and plasma lipid phenotypes in GK and $\mathrm{BN}$ rats

The regulation of lipid metabolism and glycaemia was initially investigated in rats of the parental strains used to derive the genetic cross. The concentration of plasma glucose, phospholipids, triglycerides and total, HDL and VLDL cholesterol was significantly more elevated in male and female GK rats than in sex-matched BN controls (Table 1). In contrast, LDL cholesterol levels were significantly lower in GK rats than in $\mathrm{BN}$ rats. These differences were again consistent in males and females. In both BN and GK rats, the concentration of plasma phospholipids, triglycerides and total, HDL and VLDL cholesterol was more elevated in females than in males, whereas the level of plasma LDL cholesterol was lower in females than in males. Sex-related differences were statistically significant for all lipid variables in the GK strain, whereas only plasma phospholipids and LDL and VLDL cholesterol levels were significantly different between male and female rats of the BN strain.

Phenotype analysis in GK $\times$ BN F1 and $\mathrm{F} 2$ rats

To evaluate the inheritance of these phenotypic features in a cross between GK and BN rats, we then analysed the distribution of plasma lipid values in a subset of randomly chosen heterozygous rats of the F1 generation and in all F2 hybrids. Plasma lipid and glucose values in the F1 progeny of the $\mathrm{GK} \times \mathrm{BN}$ cross were generally between those of the parental strains. However, LDL cholesterol levels in male and female F1 rats were similar to those in sex-matched GK rats (Table 1). As observed in parental strains, rats of the F1 generation showed significant sex-related differences in plasma lipid values with the exception of VLDL cholesterol concentrations, which were similar in male and female F1 rats. The existence of maternal influences in F1 rats of the two reciprocal crosses was not tested.

In the F2 hybrids, plasma phospholipid and HDL and LDL cholesterol concentrations were broadly distributed between the mean values of these variables in the two parental strains (Fig. 1). In contrast, the distribution of total cholesterol in the F2 hybrids was shifted towards the BN values and the means of this trait were very similar in the $\mathrm{F} 2$ and $\mathrm{BN}$ rats. Unexpectedly, plasma triglyceride and VLDL cholesterol concentrations were significantly lower in F2 hybrids than in $\mathrm{BN}$ and GK rats. The distribution of each phenotype in males and females was generally similar. With the exception of plasma triglyceride, sex-related differences in phenotype means followed the same pattern as previously noted in rats of the parental strains and the F1 generation. 
Table 1 Plasma lipid and glucose concentrations in 2-month-old BN, GK and GK $\times$ BN F1 rats

\begin{tabular}{|c|c|c|c|c|}
\hline & & $\mathrm{BN}$ & $\mathrm{GK}$ & $\mathrm{GK} \times \mathrm{BN}$ F1 \\
\hline \multirow[t]{2}{*}{ Phospholipids (mmol/1) } & Males & $1.08 \pm 0.03(14)$ & $1.72 \pm 0.08(6)^{*}$ & $1.33 \pm 0.03$ \\
\hline & Females & $1.37 \pm 0.06(12)^{* *}$ & $1.98 \pm 0.06(6)^{* * * * *}$ & $1.52 \pm 0.04(7)^{* * *}$ \\
\hline \multirow[t]{2}{*}{ Triglycerides (mmol/1) } & Males & $0.73 \pm 0.02(14)$ & $1.13 \pm 0.04(6)^{*}$ & $1.05 \pm 0.05(10)$ \\
\hline & Females & $0.80 \pm 0.04(12)$ & $1.81 \pm 0.16(6)^{* * * * *}$ & $1.23 \pm 0.06(7)^{* * *}$ \\
\hline \multirow[t]{2}{*}{ Total cholesterol (mmol/1) } & Males & $1.50 \pm 0.03(14)$ & $2.39 \pm 0.05(6)^{*}$ & $2.00 \pm 0.04(10)$ \\
\hline & Females & $1.62 \pm 0.06(12)$ & $2.67 \pm 0.09(6)^{* * * * *}$ & $2.12 \pm 0.05(7)^{* * *}$ \\
\hline \multirow[t]{2}{*}{ HDL cholesterol (mmol/l) } & Males & $0.70 \pm 0.03$ & $1.37 \pm 0.03(6)^{*}$ & $1.19 \pm 0.02(10)$ \\
\hline & Females & $0.79 \pm 0.03(12)$ & $1.50 \pm 0.02(6)^{* * * *}$ & $1.36 \pm 0.03(7)^{* * * *}$ \\
\hline \multirow[t]{2}{*}{ LDL cholesterol (mmol/l) } & Males & $0.37 \pm 0.03$ & $0.16 \pm 0.01(6)^{*}$ & $0.14 \pm 0.01$ \\
\hline & Females & $0.23 \pm 0.02(12)^{* *}$ & $0.12 \pm 0.01(6)^{* * * * *}$ & $0.11 \pm 0.01(7)^{* *}$ \\
\hline \multirow[t]{2}{*}{ VLDL cholesterol (mmol/1) } & Males & $0.44 \pm 0.02(14)$ & $0.86 \pm 0.03(6)^{*}$ & $0.67 \pm 0.03(10)$ \\
\hline & Females & $0.60 \pm 0.04(12)^{* *}$ & $1.00 \pm 0.05(6)^{* * * * *}$ & $0.69 \pm 0.02(7)$ \\
\hline \multirow[t]{2}{*}{ Glucose (mmol/1) } & Males & $5.37 \pm 0.22(12)$ & $9.44 \pm 0.38(6)^{*}$ & $6.74 \pm 0.20$ \\
\hline & Females & $5.53 \pm 0.23(8)$ & $10.13 \pm 0.05(6)^{*}$ & $6.58 \pm 0.14(6)$ \\
\hline
\end{tabular}

Values are means $\pm \mathrm{SE}(n)$.

${ }^{*} p<0.001$ for difference between $\mathrm{GK}$ and sex-matched $\mathrm{BN}$ rats

$* * p<0.01$

$* * * p<0.05$

$* * * * p<0.01$ for sex-related differences within groups

Pairwise Pearson correlation coefficients between each of the six logarithm-transformed phenotypes, regressed for sex effects, are reported in Table 2. Plasma phospholipids were very highly correlated $(0.815)$ with total cholesterol levels. Plasma HDL cholesterol was significantly correlated with both phospholipids and total cholesterol after adjusting for multiple testing, while triglycerides were significantly correlated with phospholipids.

The significance of differences in plasma lipid concentrations between $\mathrm{GK}$ and $\mathrm{BN}$ strains and their broad distribution in the F2 hybrids indicate that multiple strain-specific gene variants, which can be mapped to the genome by genetic linkage analysis in the F2 cross, affect these phenotypes.

\section{Genetic maps}

A genetic map was constructed using genotype data generated from the 147 markers typed in the $\mathrm{GK} \times \mathrm{BN}$ F2 hybrids (see Electronic supplementary material [ESM] Table 1). The markers spanned a total distance of $1,606 \mathrm{cM}$, which is consistent with our previously published consensus genomewide linkage map of the rat constructed in an F2 cross derived from an identical strain combination [13, 14] (ESM Table 2). The calculated average distance between loci was $10.9 \mathrm{cM}$ overall and marker order was consistent with our previously published maps [13, 14].

Quantitative trait locus analysis

Full genome-wide scans of the six lipid-lipoprotein phenotypes quantified in the $\mathrm{GK} \times \mathrm{BN}$ F2 cross are shown in ESM Fig. 1 and available through our genetic data repository (http://www.well.ox.ac.uk/rat_mapping_resources/ lipidQTL). We found strong evidence of genetic linkage between the plasma levels of phospholipids and total, HDL and LDL cholesterol and markers in three different regions, on rat chromosomes 1, 3 and 5. Despite high genome coverage, only marginal linkage to triglycerides was detected and no statistically significant linkages to VLDL cholesterol were found. We confirmed previously reported highly significant linkage between body weight and marker loci in chromosome 7 (locus Bw/gkl) [12] (data not shown).

On chromosome 1, evidence of highly significant linkage was found between the concentration of plasma phospholipids and markers located in the 12-cM telomeric end of the chromosome (maximum LOD5.6; $p=7.6 \times 10^{-6}$ ) (Fig. 2a, Table 3). This locus, denoted Plp/gkl, explains $16.7 \%$ of the variance of the level of phospholipids (Table 3). Markers in the same region exhibited evidence of genetic linkage to total cholesterol (maximum LOD4.0; $p=6.9 \times 10^{-5}$ ) and HDL cholesterol (maximum LOD3.3; $p=0.0004$ ). The QTL $P l p / g k 1$ accounts for 11.9 and $9.8 \%$ of the variance of the level of total cholesterol and HDL cholesterol, respectively. Marginal linkage was also detected between plasma triglyceride concentration and a locus $80 \mathrm{cM}$ more centromeric to Plp/gkl (maximum LOD2.9; $p=0.0014$ ). This locus was also linked to the ratio between plasma triglycerides and HDL cholesterol (maximum LOD2.9; $p=0.0010$ ), which is indicative of LDL particle size (maximum LOD2.7; $p=0.0015$ ) [22]. At these loci, GK alleles were associated with a significant increase in 

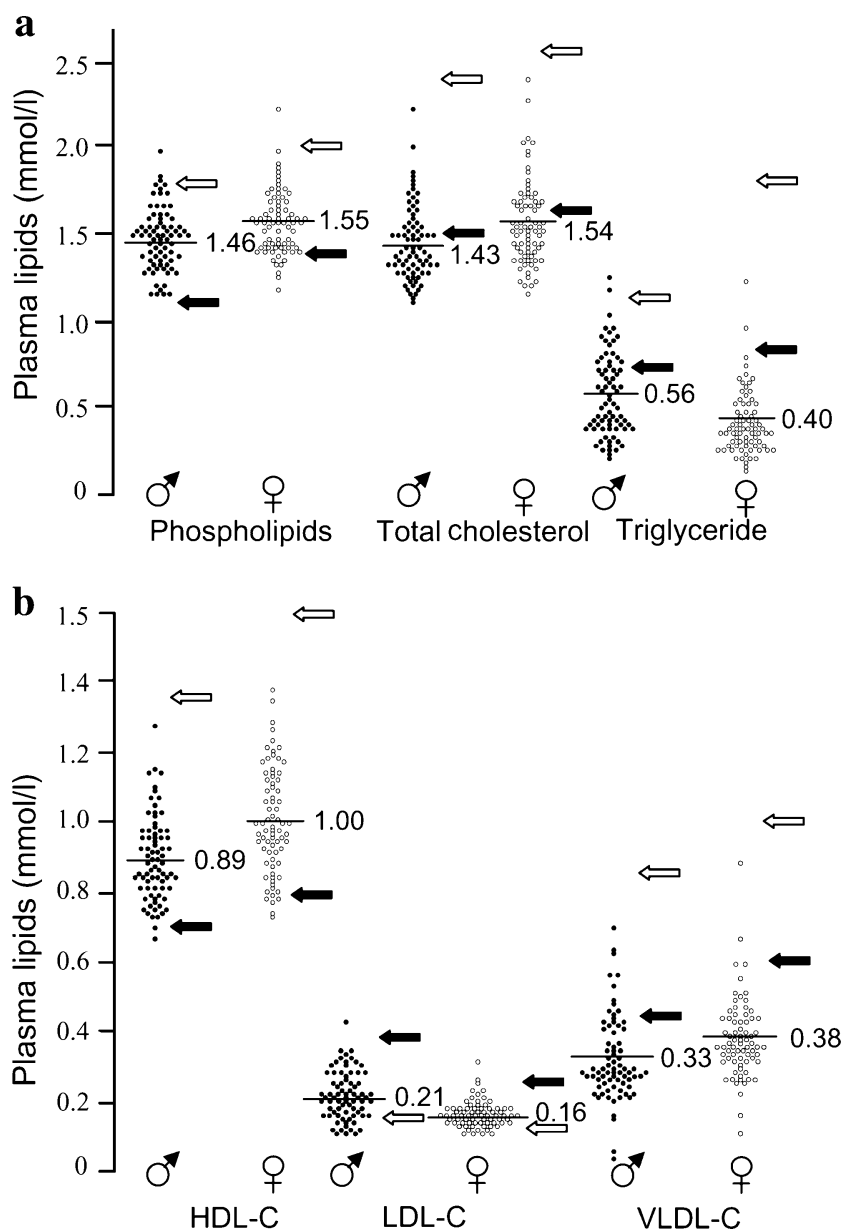

Fig. 1 Phenotype distribution of plasma lipid-lipoprotein variables in male $(\bullet)$ and female $(\bigcirc)$ hybrids of the $\mathrm{GK} \times \mathrm{BN}$ F2 cohort. Mean values of the traits in the F2 cohort are reported. White and black arrows indicate the mean values in GK and $\mathrm{BN}$ parental strains, respectively

the concentration of phospholipids, triglycerides, total cholesterol and HDL cholesterol (Table 4). The GK allelic effect on phospholipids, total cholesterol and HDL cholesterol was recessive whereas it was additive on triglycerides.

We identified in the central region of rat chromosome 3 a significant locus, denoted $L d l / g k l$, that was specifically linked to the level of plasma LDL cholesterol (maximum LOD3.9; $p=0.00016$ ) in the cross (Fig. 2b). The $95 \%$ CI of the QTL spans a 31-cM region of this chromosome. The locus $L d l / g k l$ accounts for $11.4 \%$ of the variance of LDL level in the cross (Table 3). Rats carrying the GK allele at the locus exhibited a significant decrease in LDL cholesterol and the GK allelic effect was consistent with a dominant mode of inheritance (Table 4).

Highly significant linkage was found between total cholesterol and markers localised in a 29-cM region of rat chromosome 5 (maximum LOD5.4; $p=8 \times 10^{-6}$ ) (Fig. 2c). This QTL, which we named Tchol/gk1, was also linked, but to a lesser extent, to the concentration of HDL cholesterol (maximum LOD3.3; $p=0.0012$ ). Markers in this region of chromosome 5 explain up to 16.5 and $9.6 \%$ of the variance of plasma total and HDL cholesterol, respectively, in the cross (Table 3). GK alleles at the locus contribute to a significantly increased level of total and HDL cholesterol (Table 4). The allelic affects on these phenotypes are additive.

Cross and sex-related influences in the detection of genetic linkage

The paternal or maternal origin of the GK alleles in the F2 hybrids had no influence on the lipid QTL (minimum $p=0.06$ ) (data not shown). Different effects of sex were observed for the linkages between plasma cholesterol variables and loci in chromosomes 1 and 5 (Fig. 3). While male and female hybrid rats appear to contribute equally to the significant linkage to total cholesterol in the F2 cohort at both QTLs Pl/gkl and Tchol/gkl (Fig. 3a), evidence of linkage to HDL cholesterol was due to a prominent effect in males at the locus Pl/gk1 (LOD2.3 in males and LOD0.8 in females) and a statistically significant effect in females at the locus Tchol/gkl (LOD0.7 in males and LOD3.4 in females) (Fig. 3b). A similar sexual dimorphism was observed for the QTL $L d l / g k 1$, where highly significant linkage to LDL cholesterol was found in male F2 hybrids (LOD6.0) and not in females (LOD0.9) (data not shown).

Table 2 Pearson correlations between plasma lipid phenotypes quantified in GK $\times$ BN F2 hybrids

\begin{tabular}{llllll}
\hline & PL & TG & T-C & HDL-C & LDL-C \\
\hline Phospholipids & 1 & & & & \\
Triglycerides & $0.555^{*}$ & 1 & & & \\
Total cholesterol & $0.815^{*}$ & $0.225^{*}$ & 1 & 1 & 1 \\
HDL-C & $0.661^{*}$ & 0.01 & $0.757^{*}$ & 0.093 & 0.079 \\
LDL-C & $0.250^{*}$ & 0.121 & $0.370^{*}$ & 0.109 & 1 \\
VLDL-C & $0.508^{*}$ & $0.291^{*}$ & $0.618^{*}$ & & \\
\hline
\end{tabular}

Phenotypes were log-transformed to reach normality.

$P L$ Phospholipids, $T G$ tryglycerides, $T-C$ total cholesterol, $C$ cholesterol

${ }^{*} p<0.01$ for correlation level (2-tailed) 
Fig. 2 Genetic mapping of the QTLs for plasma phospholipids (- $\square--)$, triglycerides (- - -), total cholesterol (- $\longrightarrow$, HDL cholesterol (- - - - ) and LDL cholesterol (- - ) in chromosomes 1 (Pl/gkl and $T g / g k l)$ (a), 3 (Ldl/gkl) (b) and 5 (Tcholl $g k 1)(\mathbf{c})$ in the $\mathrm{GK} \times \mathrm{BN}$ F2 hybrids. LOD scores are plotted against map distance calculated in the cross in centimorgans (cM). Permutation tests $(n=10,000)$ were used to determine statistically significant threshold of linkages $(p=0.001)$ to phenotypes, as indicated by dotted lines. Calculated statistical significance thresholds vary from 3.50 (LDL cholesterol) to 3.57 (Total cholesterol). Black bars represent the 1.5 LOD confidence intervals around the peaks of linkage for the phenotype showing the strongest evidence of linkage at the locus. The approximate 1.5 LOD confidence intervals of known GK diabetes QTLs Nidd/gkl and Nidd/gk4 are reported (a and $\mathbf{c}$, respectively)

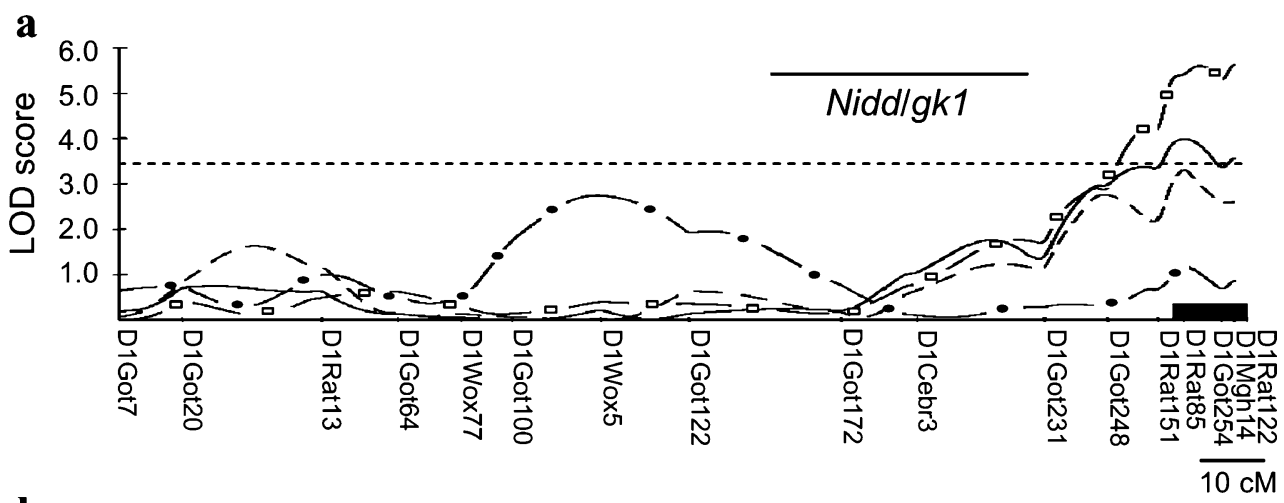

b
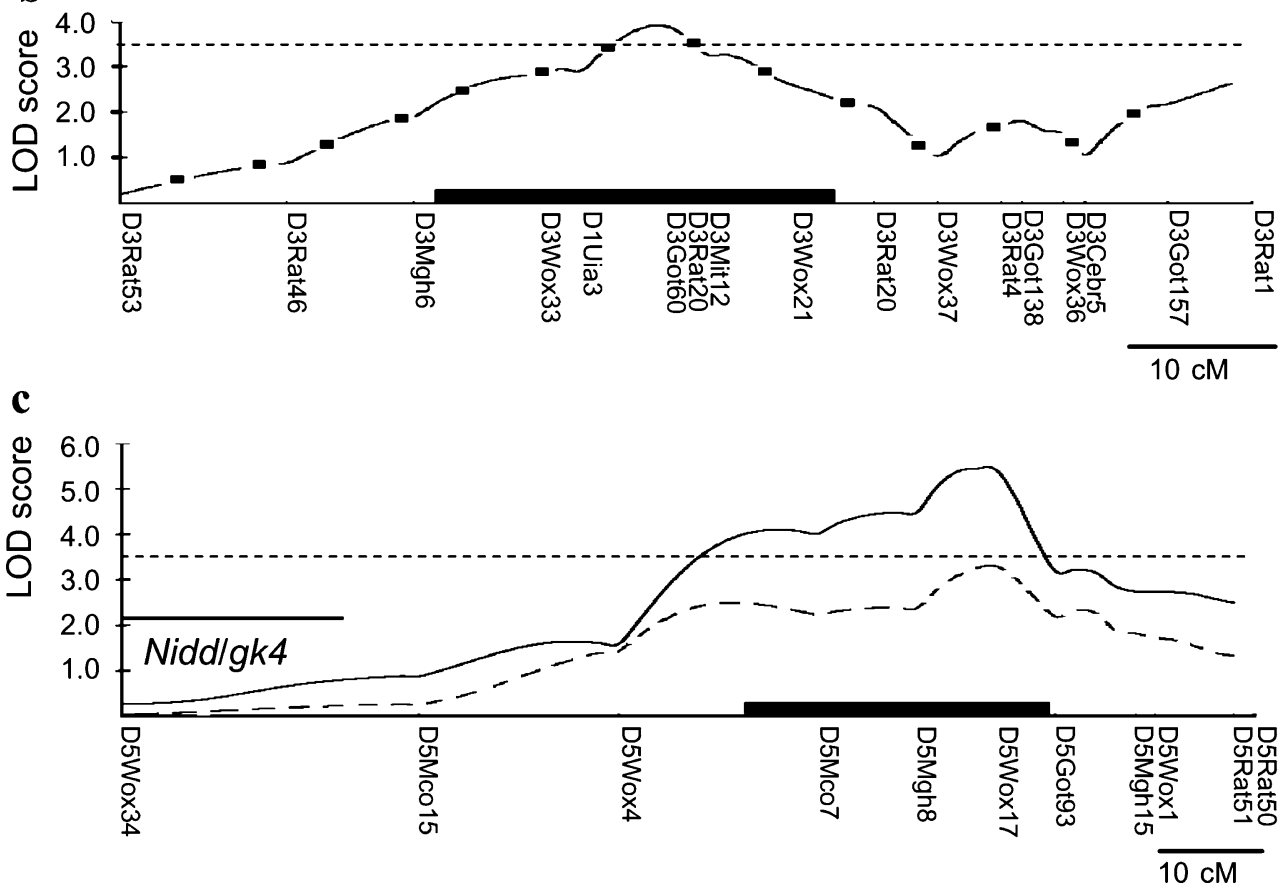

\section{Discussion}

We report the chromosomal mapping of genes regulating quantitative variations of different plasma lipid variables in an F2 cross derived from rats of the spontaneously diabetic $\mathrm{GK}$ and normoglycaemic BN strains. The characterisation of these loci in rat chromosomes 1, 3 and 5 describes a set of pathophysiological components involved in abnormal lipid metabolism in the GK rat and their genetic control in a $\mathrm{GK}-\mathrm{BN}$ strain combination. More generally, our results contribute to the finding of genetic determinants that account for mechanisms regulating cholesterol metabolism.

Owing to the complexity of genetic studies in humans, rodent models are widely used to investigate the genetic determinants of cholesterol metabolism and map to the genome gene variants underlying quantitative changes in relevant phenotypes. In rats the genetic control of plasma lipid variables has been addressed in the context of hypertension [23-25], stroke [25], obesity [26] and hyper- triglyceridaemia [27] using experimental crosses and mapping panels derived from genetically different inbred control and disease strains. Here we identify a new locus Pl/gkl that co-localises with a QTL linked to the level of plasma triglycerides and cholesterol in a cross derived from the spontaneously obese Otsuka Long-Evans Tokushima Fatty (OLETF) strain [27]. The QTL Tchol/gk1 also overlaps with loci linked to serum cholesterol levels identified in two crosses between the spontaneously hypertensive rat, stroke-prone (SHRSP) and Wistar Kyoto strains [25], and between Dahl hypertensive (SS) and Dahl normotensive (SR) rats [24]. In these strain combinations and in the GK $\times$ BN cross, Wistar Kyoto, SS and GK, alleles in this chromosomal region are associated with increased plasma cholesterol concentrations.

These strains were produced using a phenotype-driven selection of naturally occurring genetic variants isolated on the basis of glucose intolerance (GK), obesity (OLETF) or high (SHR, SS), low (SR) or normal (Wistar Kyoto) blood 
Table 3 Main characteristics of the plasma lipid-lipoprotein QTLs identified in the GK $\times$ BN F2 cross

\begin{tabular}{|c|c|c|c|c|c|c|c|c|c|}
\hline \multirow{2}{*}{$\begin{array}{l}\text { Chromosome } \\
(\mathrm{cM})^{*}\end{array}$} & \multirow{2}{*}{$\begin{array}{l}\text { Locus } \\
\text { name }\end{array}$} & \multirow{2}{*}{$\begin{array}{l}\text { Associated } \\
\text { phenotype (s) }\end{array}$} & \multirow{2}{*}{$\begin{array}{l}\text { Nearest } \\
\text { marker }\end{array}$} & \multirow{2}{*}{$\begin{array}{l}\text { Max } \\
\text { LOD }\end{array}$} & \multirow{2}{*}{$\begin{array}{l}\text { Variance } \\
(\%)\end{array}$} & \multicolumn{2}{|l|}{$95 \% \mathrm{CI}^{*}$} & \multicolumn{2}{|c|}{ Homologous regions } \\
\hline & & & & & & $(\mathrm{cM})^{*}$ & $(\mathrm{Mb})^{* *}$ & $\begin{array}{l}\text { Mouse } \\
(\mathrm{Mb})^{* *}\end{array}$ & Human cytogenetics \\
\hline \multirow[t]{3}{*}{$1(143.8)$} & $P l / g k 1$ & Phospholipids & D1Rat85 & 5.9 & 16.7 & 141-Tel & 254-Tel & $19(48-\mathrm{Tel})$ & $10 q 23-q 26$ \\
\hline & & $\begin{array}{l}\text { Total } \\
\text { cholesterol }\end{array}$ & D1Rat85 & 4.0 & 11.9 & 129-Tel & & & \\
\hline & & $\begin{array}{l}\text { HDL } \\
\text { cholesterol }\end{array}$ & D1Rat85 & 3.3 & 9.8 & 127-Tel & & & \\
\hline $1(64.9)$ & $T g / g k l$ & Triglycerides & D1Wox 5 & 2.9 & 9.6 & $50-92$ & $90-176$ & $7(32-109)$ & $\begin{array}{l}10 \mathrm{p} 11,11 \mathrm{p} 14-\mathrm{p} 15, \\
11 \mathrm{q} 13-\mathrm{q} 14,15 \mathrm{q} 11, \\
15 \mathrm{q} 25-\mathrm{q} 26,16 \mathrm{p} 13, \\
19 \mathrm{q} 12-\mathrm{q} 13\end{array}$ \\
\hline $3(65.3)$ & $L d l / g k l$ & $\begin{array}{l}\text { LDL } \\
\text { cholesterol }\end{array}$ & D3Got60 & 3.9 & 11.4 & $49-78$ & $58-126$ & $2(73-140)$ & $\begin{array}{l}2 \mathrm{p} 11-\mathrm{p} 13,2 \mathrm{q} 11-\mathrm{q} 13, \\
2 \mathrm{q} 22-\mathrm{q} 32,11 \mathrm{p} 14, \\
11 \mathrm{q} 12,15 \mathrm{q} 13-\mathrm{q} 21\end{array}$ \\
\hline \multirow[t]{2}{*}{$5(75.6)$} & Tchol/gkl & $\begin{array}{l}\text { Total } \\
\text { cholesterol }\end{array}$ & D5Wox17 & 5.4 & 16.5 & $51-80$ & $122-159$ & $4(100-139)$ & $1 \mathrm{p} 31.2,11 \mathrm{p} 32-\mathrm{p} 36$ \\
\hline & & $\begin{array}{l}\text { HDL } \\
\text { cholesterol }\end{array}$ & D5Wox17 & 3.3 & 9.6 & $43-88$ & & & \\
\hline
\end{tabular}

The permutation LOD scores (10,000 permutations) for these traits that determine statistical significance thresholds varied from 3.50 (LDL cholesterol) to 3.57 (Total cholesterol).

$m B$ Megabase

*Genetic positions of markers showing the strongest evidence of linkage to lipid variables and $95 \%$ CIs were obtained from our high-density reference rat linkage map constructed in a $\mathrm{GK} \times \mathrm{BN}$ cross [13].

**Data derived following the integration of the rat genetic map in the rat genome sequence and comparative genome analyses (http://www.well.ox.ac.uk/rat_mapping_resources) [34]. We only report physical distances in the rat, mouse and human genomes (http://www.ensembl.org/Rattus_norvegicus/) for the 95\% CI intervals of the most significant QTL.

pressure. The localisation of QTLs for intermediate phenotypes, including plasma lipids, in these strains is important for defining aetiological relationships between these traits and directly relevant disease traits (i.e. hypertension or diabetes). It is possible that identical gene variants that alter lipid regulation have been isolated in different strains by chance as part of a disease haplotype. It is also possible that these variants have been selected because they contribute to or compensate glucose intolerance and hypertension. The complexity of this situation in the context of the metabolic syndrome is illustrated by our recent findings [28]. In that study, the gene that encodes the lipid phosphatase type-II SH2-domain-containing inositol 5-phosphatase and maps to QTLs linked to hypertension in SHR and glucose intolerance in GK had an insulin-resistance-causing variant that existed in both strains.

Table 4 Correlations between genotypes at the QTLs linked to plasma lipid-lipoprotein traits in the GK $\times$ BN F2 cross

\begin{tabular}{|c|c|c|c|c|c|c|}
\hline Locus & Phenotype & $\mathrm{BN} / \mathrm{BN}$ & $\mathrm{GK} / \mathrm{BN}$ & GK/GK & $F$ & $P$ \\
\hline \multirow[t]{3}{*}{$P l / g k 1$} & Phospholipids & $1.44 \pm 0.03$ & $1.48 \pm 0.02(78)$ & $1.61 \pm 0.04$ & 6.5 & 0.0004 \\
\hline & Total-C & $1.45 \pm 0.04$ & $1.46 \pm 0.03(78)$ & $1.61 \pm 0.04$ & 5.0 & 0.003 \\
\hline & HDL-C & $0.94 \pm 0.03$ & $0.93 \pm 0.02(78)$ & $1.00 \pm 0.02$ & 3.3 & 0.02 \\
\hline$T g / g k 1$ & Triglycerides & $0.38 \pm 0.04$ & $0.48 \pm 0.03(70)$ & $0.52 \pm 0.04$ & 3.9 & 0.01 \\
\hline$L d l / g k 1$ & LDL-C & $0.22 \pm 0.01$ & $0.18 \pm 0.01(83)$ & $0.17 \pm 0.01$ & 4.3 & 0.006 \\
\hline \multirow[t]{2}{*}{ Tchol/gkl } & Total-C & $1.36 \pm 0.02(37)$ & $1.48 \pm 0.02(81)$ & $1.61 \pm 0.05$ & 11.0 & $2 \times 10^{-6}$ \\
\hline & HDL-C & $0.88 \pm 0.02$ & $0.94 \pm 0.02(81)$ & $1.01 \pm 0.03$ & 5.7 & 0.001 \\
\hline
\end{tabular}

For all phenotypes, means \pm SE $(n)$ were calculated for each genotype at the marker locus showing the strongest evidence of linkage as reported in Table 3.

$B N / B N$, Homozygous for the BN allele, $G K / B N$ heterozygous, $G K / G K$ homozygous for the GK allele, $C$ cholesterol 
Fig. 3 Sex-related influences in the detection of linkage to total (a) and HDL (b) cholesterol in rat chromosomes (Chr) 1 and 5 . Means \pm SE were calculated with values from rats homozygous for the BN allele $(\square)$, heterozygous $(\square)$ and homozygous for the GK allele ( $\square$ ) at the locus. Results from the ANOVA test are reported. Number of rats, see bars
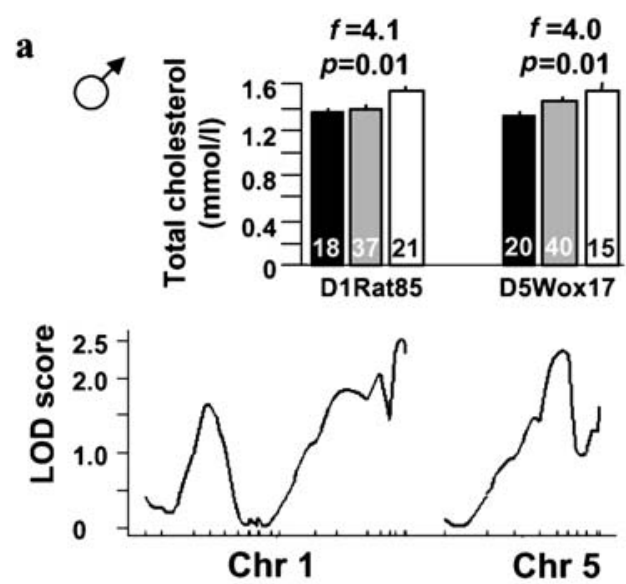

b
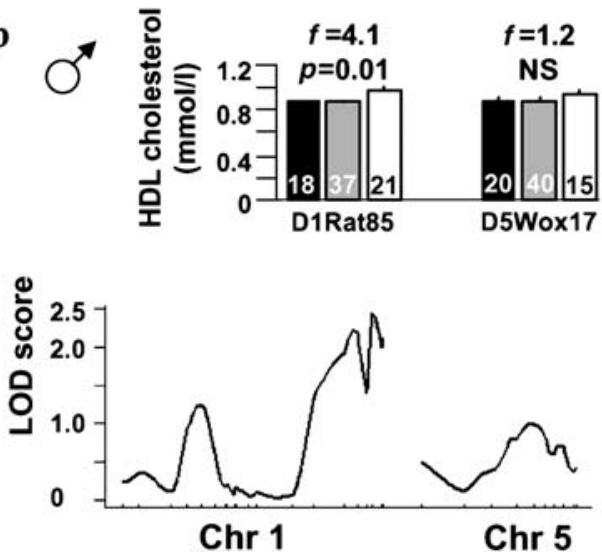
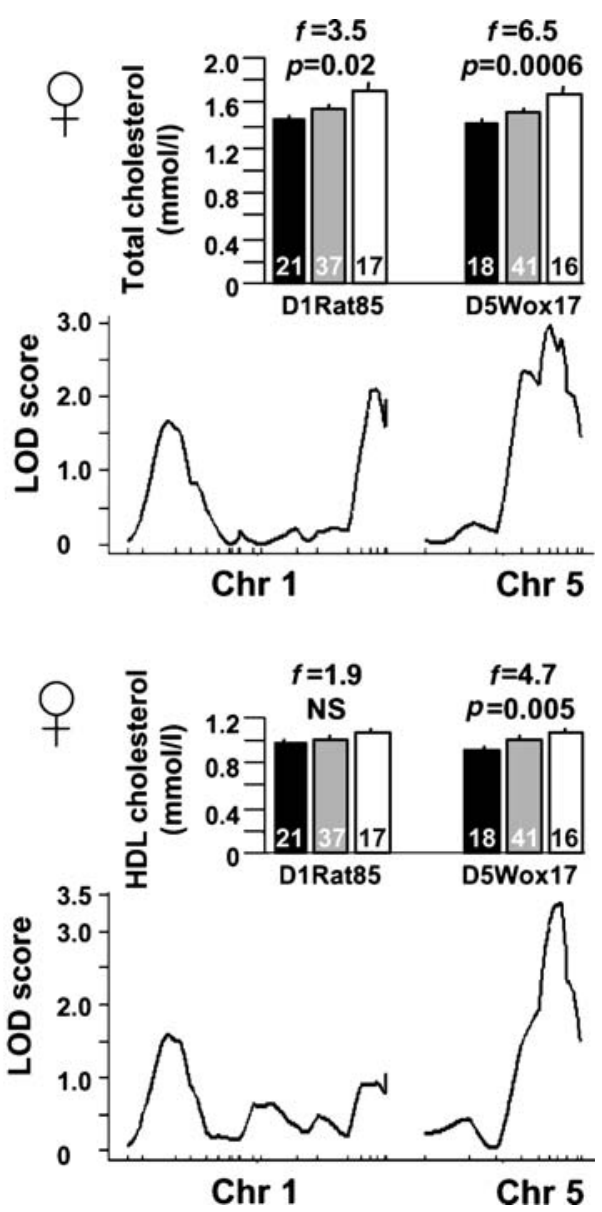

Our results provide novel pathophysiological and genetic information that further characterise the GK rat as a model of the metabolic syndrome. When compared with rats of the BN strain, GK rats show an up regulation of all categories of plasma lipids examined in this study, except LDL cholesterol. These results confirm reports of significantly elevated concentrations of plasma cholesterol and triglycerides in GK rats when compared with Wistar controls $[9,29]$. Even though the lipid QTLs reported here appear to map to different locations than previously identified GK diabetes and obesity QTLs [11, 12], we cannot rule out the possibility that the altered regulation of plasma triglycerides and HDL cholesterol in GK rats has a direct impact on diabetes severity and progression. Further genetic studies of blood pressure regulation in crosses of the GK rat will be crucial to our understanding of the aetiological relationships between pathophysiological components of the metabolic syndrome in this model.

Bearing in mind differences in lipoprotein metabolism and plasma cholesterol composition between rodents and humans [30], knowledge of relationships (synteny conservation) between rat, mouse and human genomes makes it possible to directly compare loci controlling biologically related phenotypes in these species and helps translate results from rat QTL studies to human genetics. The abundant literature in the field of genetic control of plasma cholesterol in murine models, which has predominantly focused on the regulation of HDL cholesterol in response to dietary changes, has been recently reviewed [31]. It emerges that the regulation of HDL cholesterol involves multiple genes and that there is increasing evidence of QTL replication in different crosses and strain combinations. We found little overlap between QTLs for HDL cholesterol in mice and the lipid QTL mapped in the $\mathrm{GK} \times \mathrm{BN}$ cross. Based on rat-human synteny conservation data, segments of the QTL $T g / g k 1$ are homologous to loci linked to plasma triglyceride levels in human chromosomes $15 \mathrm{q} 11-\mathrm{q} 13$ in a Mexican American population [32] and 19q13.2 [33].

Sex-related influences affect to various degrees the lipid QTLs reported here. Even though QTLs are statistically significant in the entire F2 cohort when phenotypes are corrected for sex, Tchol/gkl appears specific to females, whereas $P l / g k l$ and $L d l / g k l$ are due to an exclusive or more prominent effect in males. Such sexual dimorphism in the detection and significance of plasma lipid QTLs has been reported in rats [25] and humans [34]. Interactions between autosomal QTLs and sex-linked genes can explain this phenomenon. However, we recently reported sexual dimor- 
phism of chromosome 1 QTLs linked to glucose tolerance, insulin secretion and plasma lipid levels in an F2 cross between BN.GK congenics, where chromosome $\mathrm{X}$ alleles are fixed for BN genotypes in all hybrids, suggesting that hormonal influences on gene regulation or other variables that are correlated with sex may play an important role [35].

Rat genome annotations (http://www.ensembl.org/ Rattus_norvegicus) and comparative genomics [13] provide information on candidate genes at the GK lipid QTLs. The QTL Tchol/gk1 contains numerous candidates, including the sterol carrier protein 2 ( $S c p 2)$ and clusters of cytochrome P450 and phospholipase A2 genes involved in the synthesis and transport of lipids [36]. A mutation found in both the GK and OLETF strains [37], which prevents the translation of a G-protein-coupled receptor (Gpr10) and is believed to cause hyperphagia, obesity and dyslipidaemia in OLETF rats, may account for at least part of the QTL Pl/ $g k 1$. As outlined above, the existence of this variant in GK and OLETF rats supports its aetiological role in diabetes and obesity in these strains.

Further studies in congenic strains containing regions of GK QTLs introgressed on to the genetic background of the BN strain [38] are required to validate the lipid QTLs reported here and carry out the identification of the underlying gene variant(s). Congenics allow the study of phenotypic consequences of selected GK alleles at a specific locus in a different genetic context than that of a hybrid $\mathrm{GK} \times \mathrm{BN}$ F2 population, because $\mathrm{GK}$ variants throughout the genetic background, including at GK diabetes QTLs, are eliminated. Resulting changes in the balance between $\mathrm{GK}$ and $\mathrm{BN}$ alleles in congenics may in turn unmask phenotypic effects of some gene variants at the targeted locus. This may explain the effect of GK alleles in chromosome 8 on plasma lipid up-regulation, which we detected in BN.GK congenic rats [38] but do not replicate here in the $\mathrm{GK} \times \mathrm{BN}$ F2 cross. We found no significant evidence of genetic interaction (epistasis) in the F2 cross between GK alleles on chromosome 8 and $\mathrm{BN}$ alleles in the genetic background that could explain the absence of QTL replication (data not shown). Investigations in congenic strains designed for the lipid QTLs should also allow us to indirectly test the role of GK alleles on the $\mathrm{X}$ chromosome, which may account for sex-related effects on the detection and significance of the cholesterol QTLs.

In summary, we have identified several genetic loci that control the levels of plasma triglyceride, phospholipids, total cholesterol, HDL and LDL-cholesterol in the GK rat and appear to be independent of QTLs for glucose intolerance and altered insulin secretion in this strain. These QTLs represent chromosomal targets for identifying genes that participate in the complex control of lipid metabolism. They are also targets for the design of novel congenic strains, which by combining GK alleles at several selected QTLs known to cause glucose intolerance and an atherogenic profile, will provide new resources to study biological relationships between risk factors for the metabolic syndrome.

Acknowledgements This work was supported by the Wellcome Trust and a grant from the European Commission (GIFT-QLRT1999-00546). D. Gauguier holds a Wellcome Senior Fellowship in Basic Biomedical Science (057733). S. P. Wilder is a recipient of a Wellcome Prize Studentship in Bioinformatics and Statistical Genetics.

Duality of interest The authors declare that they do not have any financial interests that might conflict with this study.

\section{References}

1. DeFronzo RA, Ferrannini E (1991) Insulin resistance. A multifaceted syndrome responsible for NIDDM, obesity, hypertension, dyslipidemia, and atherosclerotic cardiovascular disease. Diabetes Care 14:173-194

2. Kahn R, Buse J, Ferrannini E, Stern M (2005) The metabolic syndrome: time for a critical appraisal. Joint statement from the American Diabetes Association and the European Association for the Study of Diabetes. Diabetologia 48:1684-1699

3. Eckel RH, Grundy SM, Zimmet PZ (2005) The metabolic syndrome. Lancet 365:1415-1428

4. Stern MP (1995) Diabetes and cardiovascular disease. The "common soil" hypothesis. Diabetes 44:369-374

5. Stern MP (2000) Strategies and prospects for finding insulin resistance genes. J Clin Invest 106:323-327

6. Cox RD, Brown SD (2003) Rodent models of genetic disease. Curr Opin Genet Dev 13:278-283

7. Portha B (2005) Programmed disorders of beta-cell development and function as one cause for type 2 diabetes? The GK rat paradigm. Diabetes Metab Res Rev 21:495-504

8. Gronholm T, Cheng ZJ, Palojoki E et al (2005) Vasopeptidase inhibition has beneficial cardiac effects in spontaneously diabetic Goto-Kakizaki rats. Eur J Pharmacol 519:267-276

9. O'Rourke CM, Davis JA, Saltiel AR, Cornicelli JA (1997) Metabolic effects of troglitazone in the Goto-Kakizaki rat, a non-obese and normolipidemic rodent model of non-insulindependent diabetes mellitus. Metabolism 46:192-198

10. Goto Y, Suzuki KI, Sasaki M, Ono T, Abe S (1988) GK rat as a model of nonobese, noninsulin-dependent diabetes. Selective breeding over 35 generations. In: Shafrir E, Renold AE (eds) Lessons from animal diabetes II. Libbey, London, pp 301-303

11. Galli J, Li LS, Glaser A et al (1996) Genetic analysis of noninsulin dependent diabetes mellitus in the GK rat. Nat Genet 12:31-37

12. Gauguier D, Froguel P, Parent V et al (1996) Chromosomal mapping of genetic loci associated with non-insulin dependent diabetes in the GK rat. Nat Genet 12:38-43

13. Wilder SP, Bihoreau MT, Argoud K, Watanabe TK, Lathrop M, Gauguier D (2004) Integration of the rat recombination and EST maps in the rat genomic sequence and comparative mapping analysis with the mouse genome. Genome Res 14:758-765 
14. Bihoreau MT, Gauguier D, Kato N et al (1997) A linkage map of the rat genome derived from three F2 crosses. Genome Res 7:434-440

15. Stam P (1995) Construction of integrated genetic linkage maps by means of a computer package: JoinMap. Plant J 5:739-744

16. Ihaka R, Gentleman R (1996) R: A language for data analysis and graphics. J Comput Graph Statist 5:299-314

17. Ury HK (1976) A comparison of four procedures for multiple comparisons among means (pairwise contrasts) for arbitrary samples sizes. Technometrics 18:89-97

18. Doerge RW, Churchill GA (1996) Permutation tests for multiple loci affecting a quantitative character. Genetics 142:285-294

19. Broman KW, Wu H, Sen S, Churchill GA (2003) R/qtl: QTL mapping in experimental crosses. Bioinformatics 19:889-890

20. Haley CS, Knott SA (1992) A simple regression method for mapping quantitative trait loci in line crosses using flanking markers. Heredity 69:315-324

21. Lander ES, Green P, Abrahamson J et al (1987) MAPMAKER: an interactive computer package for constructing primary genetic linkage maps of experimental and natural populations. Genomics $1: 174-181$

22. Boizel R, Benhamou PY, Lardy B, Laporte F, Foulon T, Halimi S (2000) Ratio of triacylglycerols to HDL cholesterol is an indicator of LDL particle size in patients with type 2 diabetes and normal HDL cholesterol levels. Diabetes Care 23:1679-1685

23. Bottger A, van Lith HA, Kren V et al (1996) Quantitative trait loci influencing cholesterol and phospholipid phenotypes map to chromosomes that contain genes regulating blood pressure in the spontaneously hypertensive rat. J Clin Invest 98:856-862

24. Herrera VL, Didishvili T, Lopez LV, Myers RH, Ruiz-Opazo N (2004) Genome-wide scan identifies novel QTLs for cholesterol and LDL levels in F2[Dahl RxS]-intercross rats. Circ Res 94: 446-452

25. Kato N, Tamada T, Nabika $T$ et al (2000) Identification of quantitative trait loci for serum cholesterol levels in stroke-prone spontaneously hypertensive rats. Arterioscler Thromb Vasc Biol 20:223-229

26. Yamasaki Y, Watanabe TK, Okuno S et al (2000) Quantitative trait loci for lipid metabolism in the study of OLETF $\mathrm{x}$ (OLETF $\mathrm{x}$ Fischer 344) backcross rats. Clin Exp Pharmacol Physiol 27: $881-886$
27. Klimes I, Weston K, Kovacs P et al (2003) Mapping of genetic loci predisposing to hypertriglyceridaemia in the hereditary hypertriglyceridaemic rat: analysis of genetic association with related traits of the insulin resistance syndrome. Diabetologia 46:352-358

28. Marion E, Kaisaki PJ, Pouillon V et al (2002) The gene INPPL1, encoding the lipid phosphatase SHIP2, is a candidate for type 2 diabetes in rat and man. Diabetes 51:2012-2017

29. Yamane M, Jiao S, Kihara S et al (1995) Increased proportion of plasma apoB-48 to apoB-100 in non-insulin-dependent diabetic rats: contribution of enhanced apoB mRNA editing in the liver. J Lipid Res 36:1676-1685

30. Oschry Y, Eisenberg S (1982) Rat plasma lipoproteins: re-evaluation of a lipoprotein system in an animal devoid of cholesteryl ester transfer activity. J Lipid Res 23:1099-1106

31. Wang X, Paigen B (2005) Genetics of variation in HDL cholesterol in humans and mice. Circ Res 96:27-42

32. Duggirala R, Blangero J, Almasy L et al (2000) A major susceptibility locus influencing plasma triglyceride concentrations is located on chromosome 15q in Mexican Americans. Am J Hum Genet 66:1237-1245

33. Elbein SX, Hasstedt SJ (2002) Quantitative trait linkage analysis of lipid-related traits in familial type 2 diabetes. Diabetes 51:528-535

34. Weiss LA, Pan L, Abney M, Ober C (2006) The sex-specific genetic architecture of quantitative traits in humans. Nat Genet 30:218-222

35. Collins SC, Wallis RH, Wilder SP et al (2006) Mapping diabetes QTL in an intercross derived from a congenic strain of the Brown Norway and Goto-Kakizaki rats. Mamm Genome 17:538-547

36. Nebert DW, Russell DW (2002) Clinical importance of the cytochromes P450. Lancet 360:1155-1162

37. Watanabe TK, Suzuki M, Yamasaki Y et al (2005) Mutated Gprotein-coupled receptor GPR10 is responsible for the hyperphagia/dyslipidaemia/obesity locus of Dmo1 in the OLETF rat. Clin Exp Pharmacol Physiol 32:355-366

38. Wallis RH, Wallace KJ, Collins SC et al (2004) Enhanced insulin secretion and cholesterol metabolism in congenic strains of the spontaneously diabetic (type 2) Goto Kakizaki rat are controlled by independent genetic loci in rat chromosome 8 . Diabetologia 47:1096-1106 